\title{
Interference in Floquet-Volkov transitions
}

\author{
Sang Tae Park ${ }^{*}$ \\ Arthur Amos Noyes Laboratory of Chemical Physics, California Institute of Technology, Pasadena, California 91125, USA
}

(Received 24 April 2014; published 28 July 2014)

\begin{abstract}
Floquet states are signatured by pseudoeigenvalues which are discretely separated by the photon energy. Similarly, the laser-assisted photoemission effect (LAPE) induces electron-photon energy exchange (with momentum change), and also results in a discrete energy distribution. Both effects result from coherent interactions of electrons and photons. Here, we investigate the coherent interference between a Floquet state and the LAPE effect.
\end{abstract}

DOI: 10.1103/PhysRevA.90.013420

PACS number(s): 32.80.Rm, 79.60.-i, 73.20.At

\section{INTRODUCTION}

Floquet theory [1] predicts that when the Hamiltonian is periodic in time, such as interaction with light, the solution of a time-dependent Schrödinger or Dirac equation is given by a periodic pseudoeigenfunction multiplied by a temporal evolution at a characteristic frequency [2]. Since the pseudoeigenvector is periodic in the light oscillation period, the pseudoeigenvalues become discrete multiples of photon energy around the characteristic energy, which is the signature of the "Floquet state."

Similar to Floquet states, a photoelectron may acquire additional photon energies and necessary momenta, and this phenomenon is called the laser-assisted photoemission effect (LAPE) $[3,4]$. It has been theoretically investigated by many authors [5-8], invoking the Volkov solution [9] which is an exact solution of the Klein-Gordon equation for a free electron in an infinite planar electromagnetic wave and, therefore, a special case of Floquet states for a free electron. LAPE may obscure the intrinsic dynamics of bound electrons (see below), but can be distinguished from the intrinsic dynamics by careful investigation [8].

Both the Floquet state and LAPE effect are fundamental and coherent phenomena that result from electromagnetic interactions of electrons, and they both result in multiple energy states separated by the photon energy. In order to photoemit Floquet states when studying them in photoelectron spectroscopy (PES), the photoionization laser needs to be applied while the pump laser is inducing the (instantaneous) Floquet states. The presence of the pump laser then would inevitably cause the LAPE effect simultaneously, resulting in redistribution of the side band intensity. Recently, Wang et al. investigated the photoemission spectra of Bloch-Floquet states [10] and observed the avoided crossing and side band which are the signature of Floquet states in a massless Dirac system. In particular, they found that the momentum dependence of the side band intensity is stronger for the electron momentum perpendicular to the light incidence plane than for the parallel momentum. They argued that this finding is the opposite of the LAPE theory prediction, and consequently their observation must be pure Floquet states.

Here, we will demonstrate that not only do both effects occur, but also they coherently interfere, such that the observed momentum dependence is a direct consequence

\footnotetext{
*stpark@caltech.edu
}

of the interference between a (weak) Floquet state and a (strong) LAPE effect. We will formulate the amplitudes of the Floquet pseudoeigenstate and LAPE wave function, and their coherent interference during the photoemission. The theoretical result and numerical simulations are then compared to the experimental observation.

\section{THEORY}

\section{A. Floquet pseudoeigenstate}

The theory of the Floquet state of the massless twodimensional (2D) Dirac system was given by Zhou and $\mathrm{Wu}$ [11]. The electric field perpendicular to the electron momentum only causes state mixing via the Rabi oscillation (see Appendix A 3), and consequently an avoided crossing, but the side band amplitudes are essentially determined by the parallel component (see Appendix A 2), except for the further splitting of mixed states at avoided-crossing points. Therefore, we only consider the parallel component here to express the (unmixed) Floquet amplitude, and ignore state mixing due to the perpendicular component. The final Floquet state, if needed, can be expressed by a mixture of unmixed pseudoeigenstates, shifted by band-gap splitting (see Appendix A).

For the electric field parallel to the electron momentum, the Hamiltonian matrix becomes diagonal and therefore the characteristic angular frequency simply becomes that of the unperturbed state, and the pseudoeigenvector is proportional to the unperturbed eigenvector (see Ref. [11], Appendix B). Here, we choose the vector potential to be given by

$$
\mathbf{A}(t)=\hat{\mathbf{x}} A_{2} \cos \theta_{\mathbf{E}} \cos \omega_{p} t+\hat{\mathbf{y}} A_{2} \sin \theta_{\mathbf{E}} \sin \omega_{p} t,
$$

where $\omega_{p}$ is the light angular frequency and $\theta_{\mathbf{E}}$ is the ellipticity factor. For simplicity, we ignore the spatial dependence of the vector potential. The parallel component becomes $A^{\|}=$ $A_{x} \cos \theta_{\mathbf{k}}+A_{y} \sin \theta_{\mathbf{k}}$, where $\theta_{\mathbf{k}}=\tan ^{-1}\left(\frac{k_{y}}{k_{x}}\right)$, and the temporal evolution of the Floquet state (see Appendix A 2) becomes

$$
\Psi_{b}^{F}(t)=\Psi_{\lambda}^{\xi}(t) \sum_{m=-\infty}^{+\infty} e^{i m \phi^{\|}} J_{m}\left(\lambda \beta^{\|}\right) e^{-i m \omega_{p} t},
$$

where $\Psi_{\lambda}^{\xi}(t)=\hat{\psi}_{\lambda}^{\xi}(\mathbf{k}) e^{i \mathbf{k} \cdot \mathbf{r}} e^{-i \lambda \omega_{0} t}$ is the unperturbed eigenstate wave function, $\omega_{0}=v_{F} k$ is the unperturbed energy, $\beta=\frac{e v_{F} A_{2}}{\hbar \omega_{p}}$ is the total Floquet (interaction) parameter, $\beta^{\|}=\beta \sqrt{\cos ^{2} \theta_{\mathbf{E}} \cos ^{2} \theta_{\mathbf{k}}+\sin ^{2} \theta_{\mathbf{E}} \sin ^{2} \theta_{\mathbf{k}}}$ is its parallel component, and $\phi^{\|}=\tan ^{-1}\left(\tan \theta_{\mathbf{E}} \tan \theta_{\mathbf{k}}\right)$, such that 
(a) Floquet

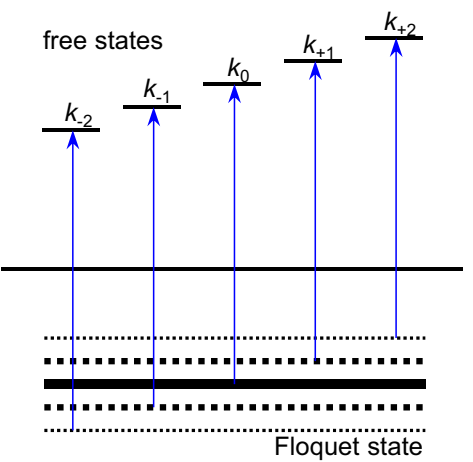

(b) LAPE

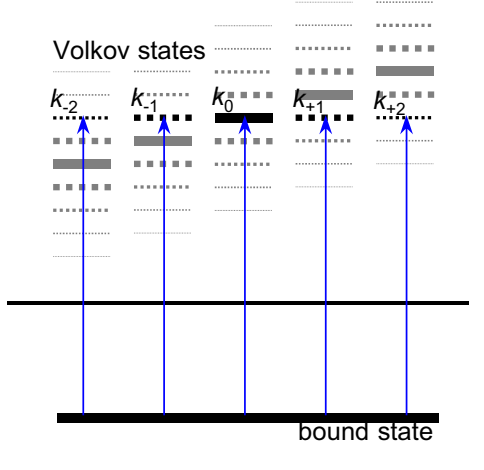

(c) Floquet/LAPE

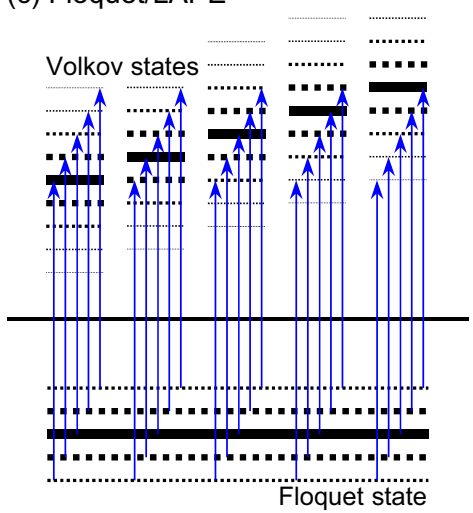

FIG. 1. (Color online) Schematic of Floquet-Volkov transitions where pseudoeigenvalues of Floquet and Volkov states enable multiple transition pathways despite energy conservation. $k_{n}$ indicates the $z$ component of the momentum of each state. Note that Volkov states collapse to free states at detection.

$\beta^{\|} e^{i \phi^{\|}}=\beta\left(\cos \theta_{\mathbf{E}} \cos \theta_{\mathbf{k}}+i \sin \theta_{\mathbf{E}} \sin \theta_{\mathbf{k}}\right)$. We note that Eq. (2) is a Volkov-like wave function.

Therefore, the Floquet coefficients become $b_{m}^{\lambda} \equiv$ $e^{i m \phi^{\|}} J_{m}\left(\lambda \beta^{\|}\right)$. For linear polarization $\left(\theta_{\mathbf{E}}=0\right), \phi^{\|}=0$ (or $\pi$ when $\left.\cos \theta_{\mathbf{k}}<0\right), \beta^{\|}=\beta\left|\cos \theta_{\mathbf{k}}\right|$, and $b_{m}^{\lambda}=J_{m}\left(\lambda \beta \cos \theta_{\mathbf{k}}\right)$. For circular polarization $\left(\theta_{\mathbf{E}}=\frac{\pi}{4}\right), \phi^{\|}=\theta_{\mathbf{k}}, \beta^{\|}=\frac{\beta}{\sqrt{2}}$, and $b_{m}^{\lambda}=e^{i m \theta_{\mathbf{k}}} J_{m}\left(\lambda \frac{\beta}{\sqrt{2}}\right)$. The photoionization of a Floquet state will result in multiple states with different energies and corresponding momenta; see Fig. 1(a).

\section{B. LAPE: Bound-Volkov transition}

Here, we adopt the "bound-Volkov transition" picture [5,12] and invoke Volkov solutions for unoccupied vacuum states, to which a bound state is photoionized; see Fig. 1(b). In this picture, we consider multiple photoionization pathways from a bound state $\left(\Psi_{b}\right)$ to many Volkov pseudoeigenstates $\left(\Psi_{n}^{V}\right)$ of different momenta $\left(\hbar k_{n}\right)$ and corresponding characteristic energies $\left(\hbar \omega_{n}\right)$, whose $j$ th pseudoeigenvalue $\left(\hbar \omega_{n, j}\right)$ coincides with the excess photon energy $\left(\hbar \omega_{e} \equiv E_{\mathrm{UV}}-W_{b}\right)$. This is equivalent to the allowed coupling between Floquet states when the pseudoeigenvalues of lower and upper states are apart by the photon energy even though the characteristic energies are not resonant (see Appendix A 3).

We ignore the (small) transverse components of the electric field and the photoelectron momentum, and therefore only consider the longitudinal component of the vector potential [13] here:

$$
\mathbf{A}(t)=\hat{\mathbf{z}} A_{1} \cos \omega_{p} t .
$$

Ignoring the ponderomotive term for simplicity [14], the nonrelativistic Volkov state [15] of the momentum $k$ becomes

$$
\Psi_{k}^{V}(t)=\Psi_{k}(t) \sum_{j=-\infty}^{+\infty} J_{j}(\alpha) e^{-i j \omega_{p} t}
$$

where $\alpha=\frac{e \mathbf{v} \cdot \mathbf{A}_{1}}{\hbar \omega_{p}}, \mathbf{v}=\hat{\mathbf{z}} \frac{\hbar k}{m_{e}}$, and $\Psi_{k}(t)=e^{i(k z-\omega t)}$ is the free electron wave function. We define the Volkov coefficients [16], $a_{j} \equiv J_{j}(\alpha)$.

Following the framework given in Refs. [5,17], with Eq. (4) and the rotating-wave approximation, the Born approximation of the scattering matrix element [18] becomes

$$
\begin{aligned}
S_{k b}^{B}-1 & =-\frac{i}{\hbar} \int_{-\infty}^{+\infty} d t\left\langle\Psi_{k}^{V}(t)\left|\frac{\hat{V}_{s} e^{-i \omega_{s} t}}{2}\right| \Psi_{b}(t)\right\rangle \\
& =-\frac{i}{\hbar}\left\langle k\left|\frac{\hat{V}_{s}}{2}\right| b\right\rangle \int_{-\infty}^{+\infty} d t \sum_{j} a_{j}^{\dagger} e^{i\left(\omega+j \omega_{p}-\omega_{b}-\omega_{s}\right) t} \\
& =-\frac{i}{\hbar} H_{k b} \sum_{j} a_{j}^{\dagger} 2 \pi \delta\left(\omega-\omega_{0}+j \omega_{p}\right),
\end{aligned}
$$

where $\hat{V}_{s}$ and $\omega_{s}$ are of photoionization, $H_{k b}=\left\langle k\left|\frac{\hat{V}_{s}}{2}\right| b\right\rangle$, and $\delta$ is the Dirac delta function [19]. Since $\omega=\frac{\hbar}{2 m} k^{2}$, Eq. (5) is only significant at discrete values, $\omega_{n}$ and $k_{n}$, with $n=-j$, where $\omega_{n}=\omega_{e}+n \omega_{p}, \omega_{0} \equiv \omega_{s}+\omega_{b}, k_{n} \approx k_{0}+n \Delta k, k_{0}=$ $\sqrt{\frac{2 m}{\hbar} \omega_{0}}$, and $\Delta k \equiv \frac{\omega_{p}}{v_{0}}$, such that the transition matrix elements (see Appendix C) become

$$
T_{n b}^{B}=H_{n b} a_{-n}^{\dagger},
$$

where $H_{n b}=\left\langle k_{n}\left|\frac{\hat{V}_{s}}{2}\right| b\right\rangle$. Since the resonance condition dictates that only the $j$ th Volkov expansion of the $n$th state contributes, where $j=-n$, the amplitude of transition to $\Psi_{n}^{V}$ is determined by its Volkov coefficient for the $(-n)$ th expansion, $a_{-n}$ [20]. Since $a_{-n}^{\dagger}=J_{-n}(\alpha)=(-1)^{n} J_{n}(\alpha)=(-1)^{n} a_{n}$, the photoelectron state becomes (see Appendix C)

$$
\begin{aligned}
\Psi^{B}(z, t) & =\frac{1}{2 \pi} \int_{-\infty}^{+\infty} d k S_{k i} \Psi_{k}^{V}(t) \\
& =-\frac{i}{\hbar} \int_{-\infty}^{+\infty} d k \sum_{n} T_{k b}^{B} \delta\left(\omega-\omega_{n}\right) \Psi_{k}^{V}(t) \\
& =-\frac{i}{\hbar} \sum_{n} \int_{-\infty}^{+\infty} d k T_{k b}^{B} \frac{\delta\left(k-k_{n}\right)}{v} \Psi_{k}^{V}(t) \\
& =-\frac{i}{\hbar} \sum_{n} \frac{T_{n b}^{B}}{v_{n}} \Psi_{n}^{V}(t) \approx-\frac{i}{\hbar} \frac{H_{0 b}}{v_{0}} \sum_{n}(-1)^{n} a_{n} \Psi_{n}^{V}(t),
\end{aligned}
$$

where we approximated $H_{n b} \approx H_{0 b}$ and $v_{n} \approx v_{0}$ for $\Delta k \ll k_{0}$ $\left(\omega_{p} \ll \omega_{0}\right)[6]$. Note that $T_{0 b}^{(0)}=H_{0 b}$ is the $T$ matrix element 
of photoemission without Volkov expansion $\left(a_{j}=\delta_{j}\right.$ when $\alpha=0)$. The pseudoeigenstate $\Psi_{n}^{V}$ continues to evolve with pseudoeigenvalues, and then collapses to a free state $\Psi_{n}$, with the energy $\hbar \omega_{n}$, after the laser interaction $(\alpha \rightarrow 0$ as $t \rightarrow \infty)$. Therefore, the final wave function at detection becomes

$$
\Psi(z, t) \rightarrow-\frac{i}{\hbar} \frac{H_{0 b}}{v_{0}} \sum_{n}(-1)^{n} a_{n} \Psi_{n} .
$$

Note the emergence of a phase factor of $(-1)^{n}$ associated with $\Psi_{n}$ of $k_{n}$ and $\omega_{n}$, which is different from the semiclassical model by Muller et al. [3] and the two-step model by Saathoff et al. [8].

In this picture, Volkov expansion is only important during photoemission because the pseudoeigenstates eventually collapse to free states when the dressing light disappears. Hence, only the electric field at the surface during the photoemission, not the entire spatial and temporal dependence, matters. The momentum component is provided by the photoionization process (and ultimately by the momentum distribution of the bound state via the $H_{n b}$ term). Note that $H_{k b}$ is a continuous function of the final (longitudinal) momentum [6], but the $\delta$ function dictates the energy resonance and hence a single momentum component in the photoemission of a bound state.

\section{Interference: Floquet-Volkov transition}

Now we consider the transition from a Floquet state $\Psi_{b}^{F}$ to many Volkov states $\Psi_{n}^{V}$; see Fig. 1(c). The result is similar to the Volkov-Volkov transition [21] in laser-assisted electron atomic scattering (LAES) [5,22,23]. Using Eqs. (2) and (4), the scattering matrix element becomes

$$
\begin{aligned}
S_{k b}^{B}-1 & =-\frac{i}{\hbar} \int_{-\infty}^{+\infty} d t\left\langle\Psi_{k}^{V}(t)\left|\frac{\hat{V}_{s} e^{-i \omega_{s} t}}{2}\right| \Psi_{b}^{F}(t)\right\rangle \\
& =-\frac{i}{\hbar}\left\langle k\left|\frac{\hat{V}_{s}}{2}\right| b\right\rangle \sum_{j, m} a_{j}^{\dagger} b_{m}^{\lambda} \int_{-\infty}^{+\infty} d t e^{i\left(\omega-\omega_{0}+(j-m) \omega_{p}\right) t} \\
& =-\frac{i}{\hbar} H_{k b} \sum_{n}\left(\sum_{j, m}^{m-j=n} a_{j}^{\dagger} b_{m}^{\lambda}\right) 2 \pi \delta\left(\omega-\omega_{0}-n \omega_{p}\right),
\end{aligned}
$$

where the $(j, m)$ summation is a discrete convolution,

$$
c_{n} \equiv \sum_{j, m}^{m-j=n} a_{j}^{\dagger} b_{m}^{\lambda}=\sum_{j^{\prime}, m}^{m+j^{\prime}=n} a_{-j^{\prime}}^{\dagger} b_{m}^{\lambda},
$$

and $a_{-j^{\prime}}^{\dagger}=(-1)^{j^{\prime}} a_{j^{\prime}}$. Using the property of the Bessel function [24], it becomes

$$
\begin{aligned}
c_{n} & =\sum_{j^{\prime}, m}^{m+j^{\prime}=n}(-1)^{j^{\prime}} J_{j^{\prime}}(\alpha) e^{i m \phi^{\|}} J_{m}\left(\lambda \beta^{\|}\right) \\
& =\left(\frac{\gamma}{|\gamma|}\right)^{n} J_{n}(|\gamma|),
\end{aligned}
$$

where

$$
\begin{aligned}
\gamma & =\lambda \beta^{\|} e^{i \phi^{\|}}-\alpha \\
& =\lambda \beta\left(\cos \theta_{\mathbf{E}} \cos \theta_{\mathbf{k}}+i \sin \theta_{\mathbf{E}} \sin \theta_{\mathbf{k}}\right)-\alpha,
\end{aligned}
$$

where $\alpha$ also depends on $\theta_{\mathbf{E}}$ via the Fresnel equation. The transition matrix becomes

$$
T_{n b}^{B}=H_{n b} c_{n},
$$

the photoelectron state becomes

$$
\Psi^{B}(z, t) \approx-\frac{i}{\hbar} \frac{H_{0 b}}{v_{0}} \sum_{n} c_{n} \Psi_{n}^{V}(t),
$$

and the (normalized) population of the $n$th state becomes

$$
Q_{n}=\left|c_{n}\right|^{2}=\left\{J_{n}(|\gamma|)\right\}^{2},
$$

since $\sum_{n}\left|c_{n}\right|^{2}=1$.

\section{RESULTS AND DISCUSSION}

The Fresnel equation for the incident light $(10 \mu \mathrm{m})$ with $E_{0}=8.9 \times 10^{7} \mathrm{~V} \mathrm{~m}^{-1}$ at $\theta_{i}=45^{\circ}$ and $n_{2}=4.93$ gives the reflectivity coefficients of $r_{p}=+0.558$ and $r_{s}=-0.747$. Therefore, we obtain $E_{x}=+2.8 \times 10^{7} \mathrm{~V} \mathrm{~m}^{-1}$ for $p$ polarization, and $E_{x}=+2.0 \times 10^{7} \mathrm{~V} \mathrm{~m}^{-1}$ and $E_{y}=+1.6 \times 10^{7} \mathrm{~V}$ $\mathrm{m}^{-1}$ for circular polarization. $E_{z}=+9.8 \times 10^{7}$ and $+6.9 \times$ $10^{7} \mathrm{~V} \mathrm{~m}^{-1}$ for $p$ and circular polarization, respectively, in vacuum. Note that $\left|E_{z}\right| \gg\left|E_{x}\right|$ because the incident and reflected amplitudes are additive for $E_{z}$, but subtractive for $E_{x}$ [25]. Using $v_{F}=5 \times 10^{5} \mathrm{~m} \mathrm{~s}^{-1}$, we obtain $\beta=0.59$ and $\theta_{\mathbf{E}}=0$ for $p$ polarization, and $\beta=0.54$ and $\theta_{\mathbf{E}}=$ 0.68 (slightly elliptical) for circularly polarized incident light. Assuming the excess energy of $\sim 0.7 \mathrm{eV}$ [26], we have $v_{z}=5.0 \times 10^{5} \mathrm{~m} \mathrm{~s}^{-1}$ whereas $v_{x}$ and $v_{y}$ are in the order of $<0.6 \times 10^{5} \mathrm{~m} \mathrm{~s}^{-1}$. Therefore, $\alpha$ depends little on the initial (bound) electron momentum [27], and we will use $\alpha=2.1$ and 1.5 for $p$ and circular polarizations, respectively, using only the $z$ components ( $x$ and $y$ components are much smaller, $\left.\left|v_{z} E_{z}\right| \gg\left|v_{x} E_{x}+v_{y} E_{y}\right|\right)$. Figure 2 plots the angle dependence in Eq. (15). It is readily seen that there is a destructive interference at $\theta_{\mathbf{k}}=0^{\circ}$ (along $+\hat{\mathbf{x}}$ ), and a constructive interference at $\theta_{\mathbf{k}}= \pm 180^{\circ}$ (along $-\hat{\mathbf{x}}$ ).

Figure 3 shows the energy spectra of the Floquet states (without photoionization laser) obtained fully (instead of the approximation given in Sec. II A) by the numerical method (see Appendix B), whereas Figs. 4 and 5 show the photoemission spectra from the Floquet states which are obtained

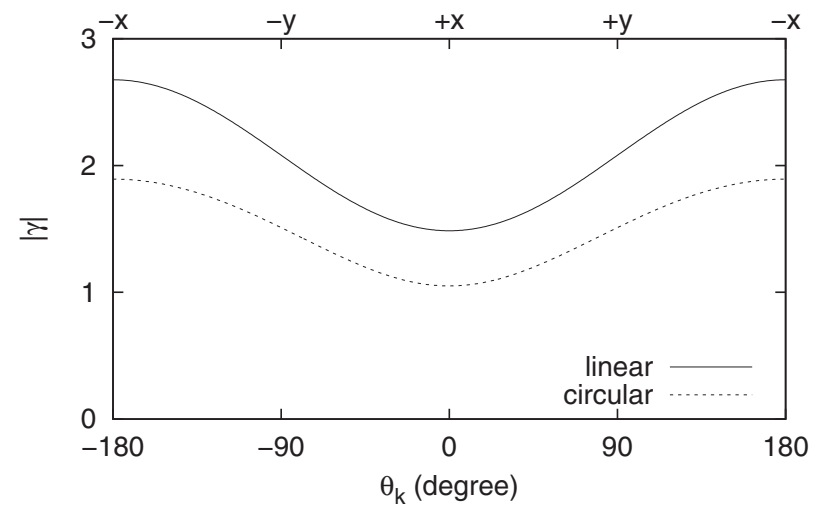

FIG. 2. Momentum direction dependence of $|\gamma|$ for $\lambda=+, \beta=$ 0.59 , and $\alpha=2.1$ and 1.5 for linear $\left(\theta_{\mathbf{E}}=0\right)$ and circular $\left(\theta_{\mathbf{E}}=\frac{\pi}{4}\right)$ polarizations, respectively. 


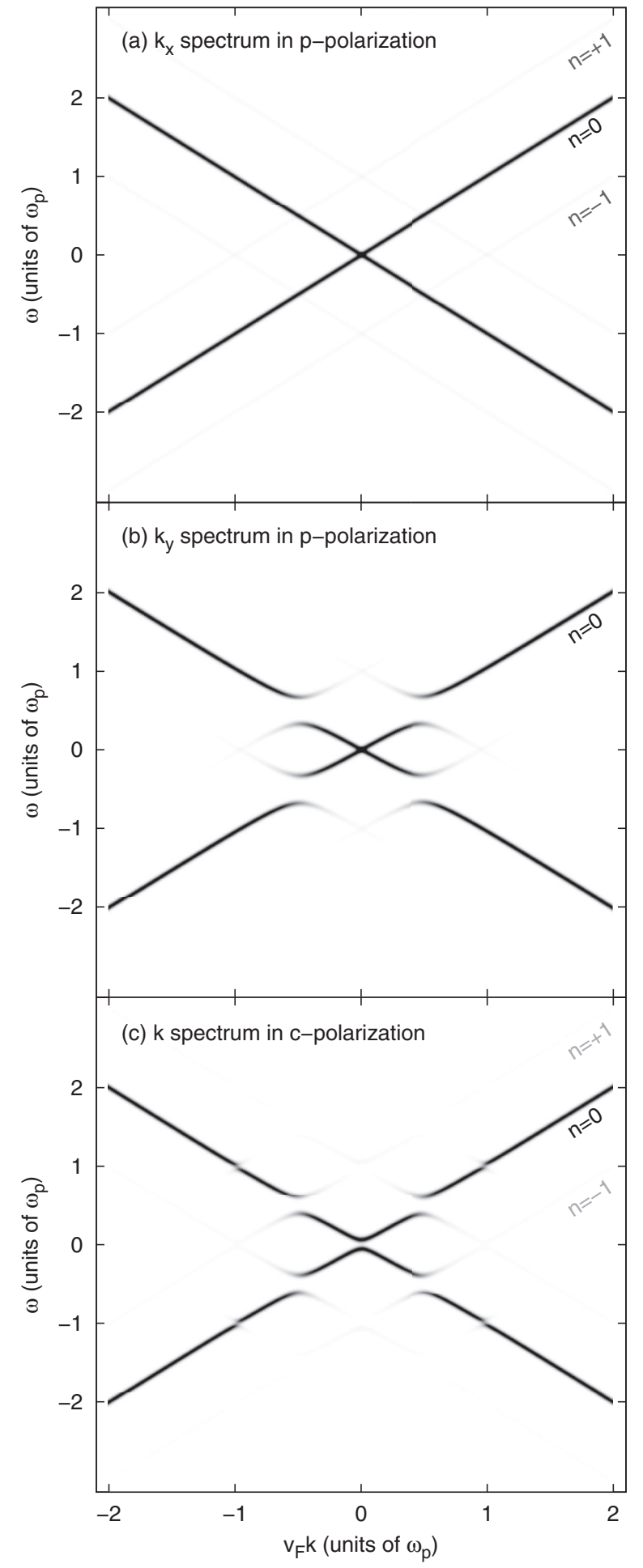

FIG. 3. Energy spectra (without UV photoionization laser) of Floquet states for $\beta=0.59$ (scaled by 0.5946 to mimic the laser profile averaging; see Appendix D). The incidence of the IR pump laser is in the $x z$ plane.

by convoluting the numerical Floquet coefficients with the LAPE amplitudes. We employed a simple scaling method (see Appendix D) and used a scaling factor of $\rho_{1}=0.5946$ to mimic the temporal and spatial overlap of the pump and probe lasers, instead of the envelope function method (see Appendix C) for simplicity. The energy spectra of Floquet

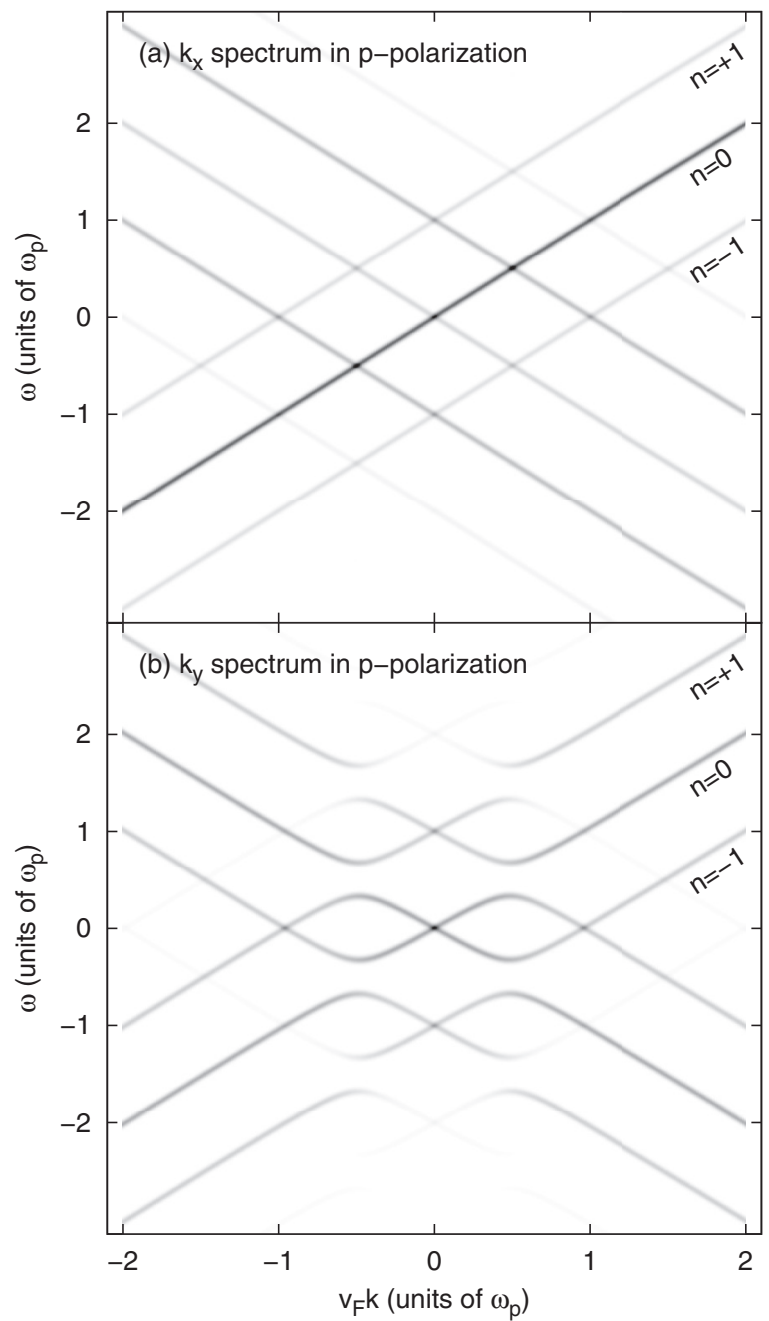

FIG. 4. Momentum-resolved photoemission spectra from Floquet states in $p$ polarization for $\theta_{\mathbf{E}}=0, \beta=0.59$, and $\alpha=2.1(\beta$ and $\alpha$ scaled by 0.5946 to mimic the laser profile averaging; see Appendix D). Additional side bands are due to LAPE.

states alone, Fig. 3, show very small side band intensities. In particular, the perpendicular momentum spectrum in linear polarization shows no side band except around $k \approx 0$, due to the lack of the electric field parallel to the electron momentum, which is responsible for Floquet side bands. In contrast, the photoemission spectra, Figs. 4 and 5, show additional and much stronger side bands (and reduced main bands) due to the very efficient LAPE effect, in addition to Floquet pseudoeigenstates.

Furthermore, due to the coherent interference between the Floquet and LAPE effects, the photoemission spectra of Floquet states depend on the electron momentum direction with respect to the incidence direction (see Figs. 4 and 5), even though the energy spectra of the Floquet states are symmetric with respect to $k=0$ and $\omega=0$ (see Fig. 3). Under $p$ polarization (Fig. 4), the LAPE effect is reduced by the Floquet state, above the Dirac point (and enhanced below) along the parallel $+\hat{\mathbf{x}}$ axis, whereas it is enhanced above (and reduced below) along the antiparallel $-\hat{\mathbf{x}}$ axis. On the other hand, along the perpendicular $\hat{\mathbf{y}}$ axis, the side band is purely by the LAPE [and no Floquet states; see Fig. 3(b)], 


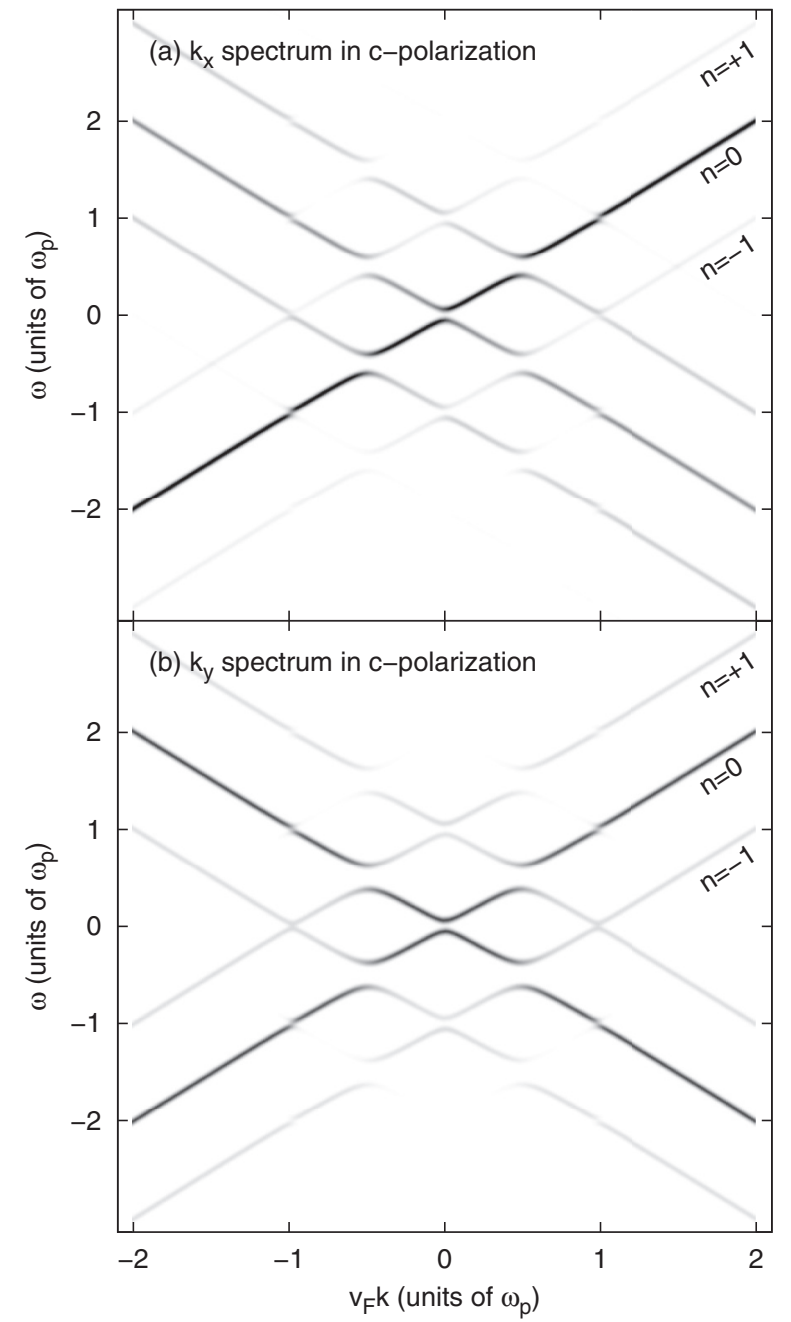

FIG. 5. Momentum-resolved photoemission spectra from Floquet states in circular polarization for $\theta_{\mathbf{E}}=0.68, \beta=0.54$, and $\alpha=$ 1.5 ( $\beta$ and $\alpha$ scaled by 0.5946 to mimic the laser profile averaging; see Appendix D). Note that the band gaps at $v_{F} k=0.5 \omega_{p}$ are not identical because the surface electric field is elliptical due to different transmittivity coefficients for $s$ - and $p$-polarization components.

and the spectrum is consequently symmetric. Under circular polarization, the parallel momentum spectrum, Fig. 5(a), shows the same asymmetric behavior as Fig. 4(a). The perpendicular momentum spectrum, Fig. 5(b), is symmetric because, in this case, the nonvanishing electric field parallel to the electron momentum is out of phase by $90^{\circ}$ with respect to $E_{z}$ which is in phase with $E_{x}$ [see Eq. (15)].

The finding above is consistent with the recent experimental observation by Wang et al. [10]. They presumably compared the side band intensities along the $+\hat{\mathbf{x}}$ axis in the $k_{x}$ spectrum $\left(k_{x}>0\right.$ and the energy above the Dirac point) and the $-\hat{\mathbf{y}}$ axis in the $k_{y}$ spectrum $\left(k_{y}<0\right.$ and the energy above the Dirac point) where the signal to noise ratio is much better due to "the coupling between the photoionization UV laser and the spin-orbit texture" [28]. They observed that the side band is much stronger in the $-\hat{\mathbf{y}}$ direction compared to that in the $+\hat{\mathbf{x}}$ direction [29]; readers are referred to Figs. 2 and 3 in Ref. [10] where the $n=+1$ band is much stronger in the $k_{y}$ spectrum than in the $k_{x}$ spectrum. The observed momentum
TABLE I. Laser-profile-weighted intensities of side bands in photoemission spectra of Floquet states above the Dirac point.

\begin{tabular}{lcccc}
\hline \hline Polarization & Momentum & $f_{0}$ & $f_{1}$ & $f_{2}$ \\
\hline$p$ & $+x$ & 0.69 & 0.15 & 0.01 \\
$p$ & $\pm y$ & 0.50 & 0.21 & 0.04 \\
$p$ & $-x$ & 0.35 & 0.24 & 0.07 \\
$c$ & $+x$ & 0.84 & 0.08 & 0.00 \\
$c$ & $\pm y$ & 0.68 & 0.15 & 0.01 \\
$c$ & $-x$ & 0.54 & 0.20 & 0.03 \\
\hline \hline
\end{tabular}

dependence is well explained by the interference between the (strong) LAPE effect and the (weak) Floquet state, as presented here (Figs. 4 and 5 here directly compare to Figs. 2 and 3 in Ref. [10], respectively; see Supplemental Material [30]), whereas the Floquet state alone (Fig. 3) does not show such a dependence. Note that the opposite reduction or enhancement would would have been observed if the comparison had been made between the $\pm \hat{\mathbf{y}}$ direction and the $-\hat{\mathbf{x}}$ direction instead. Indeed, a visual inspection seems to concur that the ratio of the side band intensity to the main band along the $-\hat{\mathbf{x}}$ axis is larger than that along the $+\hat{\mathbf{x}}$ axis (see Ref. [10], Fig. 2, panel A). In addition, the side band intensities in the Floquet-Volkov transitions simulated here (Figs. 4 and 5) are more consistent with their observations, than those of the Floquet state alone (Fig. 3), which are too weak to explain the observed intensities. This clearly shows that the LAPE is the strong and dominant effect. The simulation results with laser-profile averaging (see Appendix C) are listed in Table I.

\section{SUMMARY}

We investigated the interference between the Floquet state and LAPE effect during photoemission. The LAPE effect which depends on the vertical electric field $E_{z}$ is in phase with the incident light, whereas the phase of the Floquet state which depends on the electric field parallel to the electron momentum $E^{\|}$varies with the angle between the electron momentum and the direction of the incident light. Consequently, the combined effects exhibit an interference depending on the initial transverse momentum. The theoretical result was compared to the recent experimental observation [10] and good agreement was found.

\section{ACKNOWLEDGMENTS}

This work was supported by the National Science Foundation and the Air Force Office of Scientific Research in the Center for Physical Biology funded by the Gordon and Betty Moore Foundation. The author thanks Dr. Ahmed H. Zewail for his kind support in this project and Dr. Timothy D. Scarborough for helpful discussions.

\section{APPENDIX A: FLOQUET STATE OF MASSLESS 2D DIRAC SYSTEM}

Here, we reformulate the result of Ref. [11] using $\mathbf{A}=\hat{\mathbf{x}} A_{0} \cos \theta_{\mathbf{E}} \cos \omega_{p} t+\hat{\mathbf{y}} A_{0} \sin \theta_{\mathbf{E}} \sin \omega_{p} t \quad$ instead of $\mathbf{E}=\hat{\mathbf{x}} E_{0} \cos \theta_{E} \cos \Omega t+\hat{\mathbf{y}} E_{0} \sin \theta_{E} \sin \Omega t$ (consequently 
$\left.\mathbf{A}=-\hat{\mathbf{x}} A_{0} \cos \theta_{E} \sin \Omega t+\hat{\mathbf{y}} A_{0} \sin \theta_{E} \cos \Omega t\right)$. We choose such a vector potential for simplicity in Floquet pseudoeigenstate coefficients.

\section{Dirac Hamiltonian}

The unperturbed Hamiltonian operator is given by

$$
\hat{\mathbf{H}}_{0}=\left(\hat{H}_{0}^{\xi}\right)=\left(\begin{array}{cc}
+\hat{H}_{0} & 0 \\
0 & -\hat{H}_{0}
\end{array}\right),
$$

the component of which is given by

$$
\begin{aligned}
\hat{H}_{0}^{\xi} & =\xi \hbar v_{F}\left(\hat{k}_{x} \hat{\sigma}_{x}+\hat{k}_{y} \hat{\sigma}_{y}\right) \\
& =\xi \hbar v_{F} k\left(\begin{array}{cc}
0 & e^{-i \theta_{\mathbf{k}}} \\
e^{+i \theta_{\mathbf{k}}} & 0
\end{array}\right),
\end{aligned}
$$

where $\hat{\sigma}_{j}$ is the Pauli matrix, $\theta_{\mathbf{k}}=\tan ^{-1} \frac{k_{y}}{k_{x}}, v_{F}$ is the Fermi velocity, and $\xi= \pm$ is the valley isospin index. Then, the eigenstates are the four spinors

$$
\begin{aligned}
& \Psi_{\lambda}^{\xi=+}=e^{i \mathbf{k} \cdot \mathbf{r}} \hat{\psi}_{\lambda}^{+}(\mathbf{k})=e^{i \mathbf{k} \cdot \mathbf{r}} \frac{1}{\sqrt{2}}\left(\begin{array}{c}
1 \\
+\lambda e^{i \theta_{\mathbf{k}}} \\
0 \\
0
\end{array}\right), \\
& \Psi_{\lambda}^{\xi=-}=e^{i \mathbf{k} \cdot \mathbf{r}} \hat{\psi}_{\lambda}^{-}(\mathbf{k})=e^{i \mathbf{k} \cdot \mathbf{r}} \frac{1}{\sqrt{2}}\left(\begin{array}{c}
0 \\
0 \\
1 \\
-\lambda e^{i \theta_{\mathbf{k}}}
\end{array}\right),
\end{aligned}
$$

where $\hat{\psi}_{\lambda}^{\xi}(\mathbf{k})$ is the spinor vector and $\lambda= \pm$ is the band index. The Hamiltonian matrix for the eigenstates becomes

$$
H_{0}^{\xi}=\hbar v_{F} k\left(\begin{array}{cc}
+1 & 0 \\
0 & -1
\end{array}\right)
$$

The perturbation term is given by

$$
\begin{aligned}
\hat{H}^{\prime \xi} & =-q v_{F}\left(A_{x} \hat{\sigma}_{x}+A_{y} \hat{\sigma}_{y}\right) \\
& =-q v_{F}\left(\begin{array}{cc}
0 & A_{x}-i A_{y} \\
A_{x}+i A_{y} & 0
\end{array}\right),
\end{aligned}
$$

where $q=-e$ is the electron charge, $A_{x}=A_{2} \cos \theta_{\mathbf{E}} \cos \omega_{p} t$, and $A_{y}=A_{2} \sin \theta_{\mathbf{E}} \sin \omega_{p} t$. The perturbation matrix for the unperturbed eigenstates becomes

$$
H^{\prime \xi}=-q v_{F}\left(\begin{array}{cc}
A^{\|} & i A^{\perp} \\
-i A^{\perp} & -A^{\|}
\end{array}\right),
$$

where

$$
\left(\begin{array}{c}
A^{\|} \\
A^{\perp}
\end{array}\right)=\left(\begin{array}{cc}
\cos \theta_{\mathbf{k}} & \sin \theta_{\mathbf{k}} \\
-\sin \theta_{\mathbf{k}} & \cos \theta_{\mathbf{k}}
\end{array}\right)\left(\begin{array}{l}
A_{x} \\
A_{y}
\end{array}\right)=\mathbf{R}^{-\theta_{\mathbf{k}}}\left(\begin{array}{c}
A_{x} \\
A_{y}
\end{array}\right),
$$

which become

$$
\begin{aligned}
A^{\|} & =A_{2} \cos \theta_{\mathbf{E}} \cos \theta_{\mathbf{k}} \cos \omega_{p} t+A_{2} \sin \theta_{\mathbf{E}} \sin \theta_{\mathbf{k}} \sin \omega_{p} t \\
& =A_{2} \sqrt{\frac{1+\cos 2 \theta_{\mathbf{E}} \cos 2 \theta_{\mathbf{k}}}{2}} \cos \left(\omega_{p} t-\phi_{\|}\right), \\
A^{\perp} & =-A_{2} \cos \theta_{\mathbf{E}} \sin \theta_{\mathbf{k}} \cos \omega_{p} t+A_{2} \sin \theta_{\mathbf{E}} \cos \theta_{\mathbf{k}} \sin \omega_{p} t \\
& =A_{2} \sqrt{\frac{1-\cos 2 \theta_{\mathbf{E}} \cos 2 \theta_{\mathbf{k}}}{2}} \sin \left(\omega_{p} t-\phi_{\perp}\right),
\end{aligned}
$$

where $\tan \phi_{\|}=\frac{\sin \theta_{\mathbf{E}} \sin \theta_{\mathbf{k}}}{\cos \theta_{\mathbf{E}} \cos \theta_{\mathbf{k}}}=\tan \theta_{\mathbf{E}} \tan \theta_{\mathbf{k}}$ and $\tan \phi_{\perp}=$ $\frac{\cos \theta_{\mathbf{E}} \sin \theta_{\mathbf{k}}}{\sin \theta_{\mathbf{E}} \cos \theta_{\mathbf{k}}}=\cot \theta_{\mathbf{E}} \tan \theta_{\mathbf{k}}$. For convenience, we define $\beta \equiv$ $\frac{-q v_{F} A_{2}}{\omega_{p}}, \beta^{\|}=\beta \sqrt{\frac{1+\cos 2 \theta_{\mathbf{E}} \cos 2 \theta_{\mathbf{k}}}{2}}$, and $\beta^{\perp}=\beta \sqrt{\frac{1-\cos 2 \theta_{\mathbf{E}} \cos 2 \theta_{\mathbf{k}}}{2}}$, such that

$$
H^{\prime \xi}=\hbar \omega_{p}\left(\begin{array}{cc}
+\beta^{\|} \cos \left(\omega_{p} t-\phi^{\|}\right) & +i \beta^{\perp} \sin \left(\omega_{p} t-\phi^{\perp}\right) \\
-i \beta^{\perp} \sin \left(\omega_{p} t-\phi^{\perp}\right) & -\beta^{\|} \cos \left(\omega_{p} t-\phi^{\|}\right)
\end{array}\right) .
$$

\section{Parallel component: Side bands}

We divide the Hamiltonian matrix into diagonal and offdiagonal terms as

$$
\begin{aligned}
H^{\xi}= & \hbar\left[\omega_{0}+\omega_{p} \beta^{\|} \cos \left(\omega_{p} t-\phi^{\|}\right)\right]\left\{\hat{\sigma}_{z}\right\} \\
& -\hbar \omega_{p} \beta^{\perp} \sin \left(\omega_{p} t-\phi^{\perp}\right)\left\{\hat{\sigma}_{y}\right\} \\
\equiv & {\left[H_{0}+H_{1}(t)\right]+H_{2}(t) . }
\end{aligned}
$$

For the electric field parallel to $\mathbf{k}$, the Hamiltonian $\left(H_{0}+H_{1}\right)$ is diagonal and therefore the characteristic angular frequency simply becomes that of the unperturbed state, and the pseudoeigenvector is proportional to the unperturbed eigenvector. The temporal evolution of the pseudoeigenvector is obtained by

$$
\begin{aligned}
\Psi_{\lambda}^{\xi}(t) & =\exp \left[-\frac{i}{\hbar} \int d t H_{\lambda \lambda}\right] \Psi_{\lambda}^{\xi}(0) \\
& =\exp \left\{-i \lambda\left[\omega_{0} t+\beta^{\|} \sin \left(\omega_{p} t-\phi^{\|}\right)\right]\right\} \Psi_{\lambda}^{\xi}(0) \\
& =\Psi_{\lambda}^{\xi}(0) e^{-i \lambda \omega_{0} t} \sum_{m=-\infty}^{+\infty} J_{-m}\left(-\lambda \beta^{\|}\right) e^{-i m\left(\omega_{p} t-\phi^{\|}\right)} \\
& =\Psi_{\lambda}^{\xi}(0) \sum_{m=-\infty}^{+\infty} e^{i m \phi^{\|}} J_{m}\left(\lambda \beta^{\|}\right) e^{-i\left(\lambda \omega_{0}+m \omega_{p}\right) t},
\end{aligned}
$$

using the Jacobi-Anger relation, $e^{i z \sin \theta}=\sum_{n} J_{n}(z) e^{i n \theta}$. We define the Floquet coefficients, $b_{m}^{\lambda} \equiv e^{i m \phi^{\|}} J_{m}\left(\lambda \beta^{\|}\right)$.

\section{Perpendicular component: Rabi oscillation}

Now we assume the solution is given by

$$
\Phi_{k}^{\xi}(t)=\sum_{\lambda} c_{\lambda}(t) \Psi_{\lambda}^{\xi}(t)
$$

Then, the time-dependent Dirac equation leads to

$$
\begin{aligned}
& \dot{c}_{+}=+c_{-} \omega_{p} \beta^{\perp} \sin \left(\omega_{p} t-\phi^{\perp}\right) \frac{\sum_{m} b_{m}^{-} e^{-i\left(-\omega_{0}+m \omega_{p}\right) t}}{\sum_{m} b_{m}^{+} e^{-i\left(+\omega_{0}+m \omega_{p}\right) t}}, \\
& \dot{c}_{-}=-c_{+} \omega_{p} \beta^{\perp} \sin \left(\omega_{p} t-\phi^{\perp}\right) \frac{\sum_{m} b_{m}^{+} e^{-i\left(+\omega_{0}+m \omega_{p}\right) t}}{\sum_{m} b_{m}^{-} e^{-i\left(-\omega_{0}+m \omega_{p}\right) t}},
\end{aligned}
$$

which, using the Jacobi-Anger relation, becomes

$$
\begin{aligned}
& \dot{c}_{+}=+c_{-} \omega_{p} \beta^{\perp} \sin \left(\omega_{p} t-\phi^{\perp}\right) \sum_{m} y_{m}^{-} e^{-i\left(-2 \omega_{0}+m \omega_{p}\right) t}, \\
& \dot{c}_{-}=-c_{+} \omega_{p} \beta^{\perp} \sin \left(\omega_{p} t-\phi^{\perp}\right) \sum_{m} y_{m}^{+} e^{-i\left(+2 \omega_{0}+m \omega_{p}\right) t},
\end{aligned}
$$


where $\quad y_{m}^{\lambda}=e^{i m \phi^{\|}} J_{m}\left(2 \lambda \beta^{\|}\right)$. Using $2 i \sin \left(\omega_{p} t-\phi^{\perp}\right)=$ $e^{i \omega_{p} t} e^{-i \phi^{\perp}}-e^{-i \omega_{p} t} e^{i \phi^{\perp}}$, we apply the rotating-wave approximation for $l \omega_{p} \approx 2 \omega_{0}$ where only the terms with $m_{\lambda}=\lambda l \pm 1$ survive. Namely, with $\Delta \omega_{l} \equiv 2 \omega_{0}-l \omega_{p}$ where $\omega_{0}=v_{F} k$,

$$
\begin{aligned}
& \dot{c}_{+} \approx+c_{-} \frac{\omega_{p} \beta^{\perp}}{2 i}\left(e^{-i \phi^{\perp}} y_{+l+1}^{-}-e^{+i \phi^{\perp}} y_{+l-1}^{-}\right) e^{+i \Delta \omega_{l} t}, \\
& \dot{c}_{-} \approx-c_{+} \frac{\omega_{p} \beta^{\perp}}{2 i}\left(e^{-i \phi^{\perp}} y_{-l+1}^{+}-e^{+i \phi^{\perp}} y_{-l-1}^{+}\right) e^{-i \Delta \omega_{l} t},
\end{aligned}
$$

We define $\Omega^{+} \equiv \omega_{p} \beta^{\perp}\left(e^{-i \phi^{\perp}} y_{+l+1}^{-}-e^{+i \phi^{\perp}} y_{+l-1}^{-}\right)$and $\Omega^{-} \equiv$ $-\omega_{p} \beta^{\perp}\left(e^{-i \phi^{\perp}} y_{-l+1}^{+}-e^{+i \phi^{\perp}} y_{-l-1}^{+}\right)$. Note that $\Omega^{-}=\Omega^{+\dagger}$. The solutions are

$$
\begin{aligned}
\left(\begin{array}{l}
c_{+} \\
c_{-}
\end{array}\right)= & C_{1}\left(\begin{array}{l}
c_{1}^{+} \exp \left[-i \frac{1}{2}\left(\Omega_{l}-\Delta \omega_{l}\right) t\right] \\
c_{1}^{-} \exp \left[-i \frac{1}{2}\left(\Omega_{l}+\Delta \omega_{l}\right) t\right]
\end{array}\right) \\
& +C_{2}\left(\begin{array}{l}
c_{2}^{+} \exp \left[-i \frac{1}{2}\left(-\Omega_{l}-\Delta \omega_{l}\right) t\right] \\
c_{2}^{-} \exp \left[-i \frac{1}{2}\left(-\Omega_{l}+\Delta \omega_{l}\right) t\right]
\end{array}\right),
\end{aligned}
$$

where $\Omega_{l}=\sqrt{\Omega^{+} \Omega^{-}+\Delta \omega_{l}^{2}}$ and

$$
\begin{aligned}
c_{1}^{ \pm} & =\sqrt{\frac{\Omega^{ \pm}}{\left|\Omega^{ \pm}\right|}\left(\frac{\Omega_{l} \pm \Delta \omega_{l}}{2 \Omega_{l}}\right)}, \\
c_{2}^{ \pm} & = \pm \sqrt{\frac{\Omega^{ \pm}}{\left|\Omega^{ \pm}\right|}\left(\frac{\Omega_{l} \mp \Delta \omega_{l}}{2 \Omega_{l}}\right)} .
\end{aligned}
$$

Pseudoeigenvectors become

$$
\begin{aligned}
\Phi_{1} & =\boldsymbol{\Psi}\left(\begin{array}{l}
c_{1}^{+} b^{+}(t) \\
c_{1}^{-} b^{-}(t) e^{+i l \omega_{p} t}
\end{array}\right) e^{-i\left(\frac{l \omega_{p}+\Omega_{l}}{2}\right) t} \\
& =\boldsymbol{\Psi}\left(\begin{array}{l}
c_{1}^{+} \sum_{m} b_{m}^{+} e^{-i m \omega_{p} t} \\
c_{1}^{-} \sum_{m} b_{m+l}^{-} e^{-i m \omega_{p} t}
\end{array}\right) e^{-i\left(\frac{l \omega_{p}+\Omega_{l}}{2}\right) t} \\
& =\boldsymbol{\Psi} \sum_{m}\left(\begin{array}{l}
c_{1}^{+} b_{m}^{+} \\
c_{1}^{1} b_{m+l}^{-}
\end{array}\right) e^{-i m \omega_{p} t} e^{-i\left(\frac{l \omega_{p}+\Omega_{l}}{2}\right) t}
\end{aligned}
$$

and

$$
\begin{aligned}
\Phi_{2} & =\Psi\left(\begin{array}{l}
c_{2}^{+} b^{+}(t) e^{-i l \omega_{p} t} \\
c_{2}^{-} b^{-}(t)
\end{array}\right) e^{+i\left(\frac{l \omega_{p}+\Omega_{l}}{2}\right) t} \\
& =\boldsymbol{\Psi}\left(\begin{array}{l}
c_{2}^{+} \sum_{m} b_{m-l}^{+} e^{-i m \omega_{p} t} \\
c_{2}^{-} \sum_{m} b_{m}^{-} e^{-i m \omega_{p} t}
\end{array}\right) e^{+i\left(\frac{l \omega_{p}+\Omega_{l}}{2}\right) t} \\
& =\boldsymbol{\Psi} \sum_{m}\left(\begin{array}{l}
c_{2}^{+} b_{m-l}^{+} \\
c_{2}^{2} b_{m}^{-}
\end{array}\right) e^{-i m \omega_{p} t} e^{+i\left(\frac{l \omega_{p}+\Omega_{l}}{2}\right) t},
\end{aligned}
$$

respectively, where $\quad b^{\lambda}(t)=e^{-i \lambda \beta^{\|} \sin \left(\omega_{p} t-\phi^{\|}\right)}=$ $\sum_{m} b_{m}^{\lambda} e^{-i m \omega_{p} t}, \boldsymbol{\Psi}=e^{i \mathbf{k} \cdot \mathbf{r}} \hat{\psi}$, and $\hat{\psi}=\left(\hat{\psi}_{+}^{\xi} \hat{\psi}_{-}^{\xi}\right)$. Therefore, the $m$ th Floquet coefficients for $\Phi_{1}$ and $\Phi_{2}$ are given by $\hat{\psi}\left(c_{1}^{+} b_{m}^{+}, c_{1}^{-} b_{m+l}^{-}\right)^{T}$ and $\hat{\psi}\left(c_{2}^{+} b_{m-l}^{+}, c_{2}^{-} b_{m}^{-}\right)^{T}$, respectively, where $l$ is the nearest integer to $\frac{2 v_{F} k}{\omega_{p}}$. Note that $\Phi_{2}$ near $\Phi_{1}$ is given by replacing $m$ with $m^{\prime}+l$ as

$$
\Phi_{2}=\Psi \sum_{m^{\prime}}\left(\begin{array}{l}
c_{2}^{+} b_{m^{\prime}}^{+} \\
c_{2}^{2} b_{m^{\prime}+l}^{-}
\end{array}\right) e^{-i m^{\prime} \omega_{p} t} e^{-i\left(\frac{l \omega_{p}-\Omega_{l}}{2}\right) t} .
$$

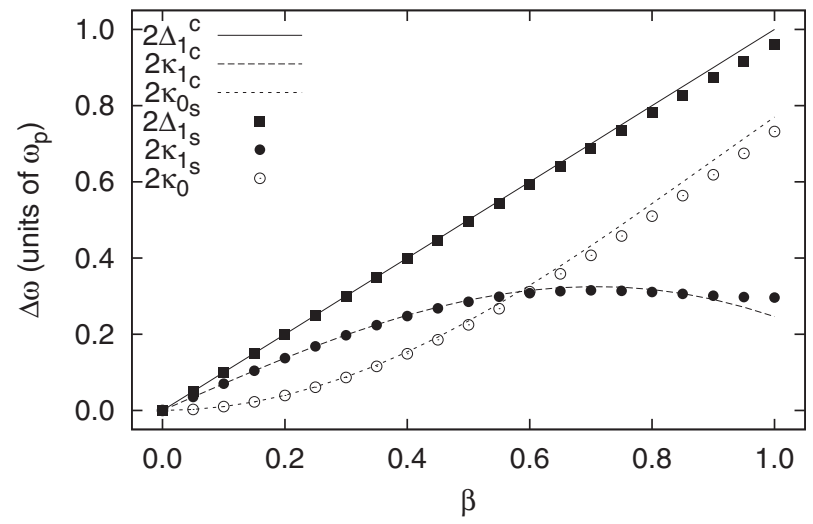

FIG. 6. Calculated (lines) and simulated (symbols) band gaps, $2 \Delta_{1}$ at $2 v_{F} k=\omega_{p}$ for the orthogonal linear polarization (solid line and square) and $2 \kappa_{l}$ at $2 v_{F} k=l \omega_{p}$ for the circular polarization (dashed and dotted lines, and circles).

Therefore, for a small $\Delta \omega_{l}$, the side band distribution by $b_{m}^{\lambda}$ is further split by twofold, and the band gap at the avoided crossing is given by $\Omega_{l}$. For a large $\Delta \omega_{l}$, mixing is negligible.

\section{Band gap}

For linear polarization $\left(\theta_{\mathbf{E}}=0, \phi^{\|}=0, \phi^{\perp}=-\frac{\pi}{2}, \beta^{\|}=\right.$ $\beta \cos \theta_{\mathbf{k}}$, and $\beta^{\perp}=-\beta \sin \theta_{\mathbf{k}}$ ), the band gap becomes

$$
\begin{aligned}
2 \Delta_{n} & =\left|\omega_{p} \beta \sin \theta_{\mathbf{k}}\left\{J_{n-1}\left(2 \beta \cos \theta_{\mathbf{k}}\right)+J_{n+1}\left(2 \beta \cos \theta_{\mathbf{k}}\right)\right\}\right| \\
& =\left|2 n \frac{J_{n}\left(2 \beta \cos \theta_{\mathbf{k}}\right)}{2 \beta \cos \theta_{\mathbf{k}}} \beta \sin \theta_{\mathbf{k}} \omega_{p}\right| \\
& =\left|n J_{n}\left(2 \beta \cos \theta_{\mathbf{k}}\right) \tan \theta_{\mathbf{k}} \omega_{p}\right| .
\end{aligned}
$$

At $n=1,2 \Delta_{1}=\left|J_{1}\left(2 \beta\left|\cos \theta_{\mathbf{k}}\right|\right) \tan \theta_{\mathbf{k}} \omega_{p}\right| \approx\left|\beta \sin \theta_{\mathbf{k}} \omega_{p}\right|$. Needless to say, at $n=0,2 \Delta_{0}=0$.

For circular polarization $\left(\theta_{\mathbf{E}}=\frac{\pi}{4}, \phi^{\|}=\phi^{\perp}=\theta_{\mathbf{k}}, \beta^{\|}=\right.$ $\beta^{\perp}=\frac{\beta}{\sqrt{2}}$, the band gap becomes

$$
\begin{aligned}
2 \kappa_{n} & =\left|\omega_{p} \frac{\beta}{\sqrt{2}}\left\{J_{n+1}(\sqrt{2} \beta)-J_{n-1}(\sqrt{2} \beta)\right\}\right| \\
& =\left|J_{n}^{\prime}(\sqrt{2} \beta) \sqrt{2} \beta \omega_{p}\right|,
\end{aligned}
$$

where $J_{n}^{\prime}$ is the derivative of the Bessel function. At $n=0$, $2 \kappa_{0}=\left|2 J_{1}(\sqrt{2} \beta) \frac{\beta}{\sqrt{2}} \omega_{p}\right| \approx \beta^{2} \omega_{p}$. Figure 6 shows calculated and simulated band gaps (see Appendix B for the numerical approach). For weak interactions, the band gaps agree well with the simulation.

\section{APPENDIX B: NUMERICAL SOLUTION OF FLOQUET STATE}

An exact solution of a Floquet state involves diagonalization of an infinite, sparse matrix which is truncated to $N M \times N M$ for $N$ states (with $N \times N$ Hamiltonian) and $M$ Floquet coefficients where truncation requires a knowledge or an educated guess of the exact solution [2]. Here, we present an alternative efficient algorithm to determine the characteristic frequencies and pseudoeigenvectors of Floquet states, using $N$ single period numerical integrations, $N \times N$ matrix diagonalization, and $N \times N$ fast Fourier transforms (FFTs) of a length of $M$. 


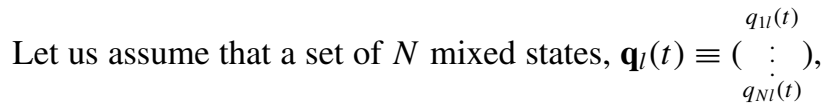
where $l=1 \cdots N$, for $N$-dimensional Hamiltonian, is known at $t=0$ and $t=\tau$, which can be obtained by numerical integration of the differential equation (time-dependent Schrödinger or Dirac equation) with the initial condition of $\mathbf{q}_{l}(0)$ for a period from 0 to $\tau$. Since $\mathbf{q}_{l}$ is in general a mixed state of the pseudoeigenstates, we can write

$$
\mathbf{q}_{l}(t)=\sum_{k} \mathbf{p}_{k}(t) e^{-i \omega_{k} t} c_{k l}
$$

which is, in matrix notation,

$$
\mathbf{Q}_{t}=\mathbf{P}_{t} \mathbf{T}_{t} \mathbf{C}
$$

where $\mathbf{Q}_{t} \equiv \mathbf{Q}(t)=\left\{\mathbf{q}_{1}(t), \mathbf{q}_{2}, \ldots, \mathbf{q}_{N}(t)\right\}=\left\{q_{j l}(t)\right\}$ is the result of numerical integration with the initial condition of $\mathbf{q}_{l}(0)$, $\mathbf{P}_{t} \equiv \mathbf{P}(t)=\left\{\mathbf{p}_{1}(t), \mathbf{p}_{2}, \cdots, \mathbf{p}_{N}(t)\right\}=\left\{p_{j k}(t)\right\}$ are the pseudoeigenvectors to be determined, and $\mathbf{C} \equiv\left\{\mathbf{c}_{1}, \mathbf{c}_{2}, \cdots, \mathbf{c}_{N}\right\}=$ $\left\{c_{k l}\right\}$ are the (constant) linear combination coefficients yet unknown. $\mathbf{T}_{t} \equiv \mathbf{T}(t)=\left\{e^{-i \omega_{k} t}\right\}$ is a diagonal matrix and describes the (characteristic) temporal evolution of the pseudoeigenvectors.

Since $\mathbf{P}_{t}$ is periodic $\left(\mathbf{P}_{\tau}=\mathbf{P}_{0}\right)$, we have, at $t=0$ and $t=\tau$,

$$
\begin{aligned}
& \mathbf{Q}_{0}=\mathbf{P}_{0} \mathbf{T}_{0} \mathbf{C}=\mathbf{P}_{0} \mathbf{C}, \\
& \mathbf{Q}_{\tau}=\mathbf{P}_{\tau} \mathbf{T}_{\tau} \mathbf{C}=\mathbf{P}_{0} \mathbf{T}_{\tau} \mathbf{C},
\end{aligned}
$$

respectively. With $\mathbf{C}=\mathbf{P}_{0}^{-1} \mathbf{Q}_{0}$, and by rearranging the other, we obtain

$$
\mathbf{Q}_{\tau} \mathbf{Q}_{0}^{-1}=\mathbf{P}_{0} \mathbf{T}_{\tau} \mathbf{P}_{0}^{-1}
$$

where the right-hand side is in the form of a diagonal matrix transformation (eigenvector decomposition). Therefore, by diagonalizing $\left(\mathbf{Q}_{\tau} \mathbf{Q}_{0}^{-1}\right)$, we can obtain the initial pseudoeigenvectors $\mathbf{P}_{0}$ and pseudoeigenvalues $\mathbf{T}_{\tau}$. Namely, the characteristic exponent becomes $\ln \left(\mathbf{T}_{\tau}\right)_{k k}=-i \omega_{k} \tau$.

Then, we obtain the pseudoeigenvector temporal evolution,

$$
\mathbf{P}_{t} \mathbf{T}_{t}=\mathbf{Q}_{t}\left(\mathbf{Q}_{0}^{-1} \mathbf{P}_{0}\right)
$$

therefore the periodic part is obtained from

$$
\mathbf{P}_{t}=\mathbf{Q}_{t}\left(\mathbf{Q}_{0}^{-1} \mathbf{P}_{0}\right) \mathbf{T}_{t}^{-1}
$$

By FFT of $\mathbf{P}_{t}$ at $t=\frac{m}{M} \tau$ where $m=0,1, \ldots, M-1$, we can determine $p_{j k, m}$.

The above procedure only requires $M$-step integration from $t=0$ to $\tau$ to determine the characteristic angular frequency, pseudoeigenvector, and $M$ Floquet coefficients. The characteristic angular frequency and pseudoeigenvectors are determined only by the (given) initial and final points. The rest (with the initial) is used for determining $M$ Floquet coefficients. Furthermore, for simplicity we can choose $\mathbf{Q}_{0}=\mathbf{I}$, namely, unit vectors of unperturbed basis, for a set of initial conditions, and then $\mathbf{Q}_{0}^{-1}$ also becomes $\mathbf{I}$.

\section{APPENDIX C: SCATTERING THEORY OF PHOTOEMISSION}

The $S$ operator is defined in the interaction picture [31] as

$$
\begin{aligned}
S & =\exp \left[-\frac{i}{\hbar} \int_{-\infty}^{+\infty} d t H\left(t^{\prime}\right)\right] \\
& =1-\frac{i}{\hbar} \int_{-\infty}^{+\infty} d t_{1} H\left(t_{1}\right)+\cdots .
\end{aligned}
$$

Only the zeroth- and first-order terms are considered in the Born approximation, such that $S^{B}-1=-\frac{i}{\hbar} \int_{-\infty}^{+\infty} d t^{\prime} H\left(t^{\prime}\right)$. Since $\langle k|H| j\rangle=\left\langle k^{(S)}\left|H_{I}^{(S)}\right| j^{(S)}\right\rangle=\left\langle k(0)\left|H_{I}^{(S)}(0)\right| j(0)\right\rangle e^{i \omega_{k j I} t}$, where $\omega_{k j I}=\omega_{k}-\omega_{j}-\omega_{I}$ for a harmonic perturbation, the $S$ matrix element becomes

$$
\begin{aligned}
S_{k j} & =\delta_{k j}-2 \pi \frac{i}{\hbar} \delta\left(\omega_{k j I}\right) T_{k j}, \\
T_{k j} & =H_{k j}^{(S)}(0)+\cdots,
\end{aligned}
$$

where $\quad H_{k j}^{(S)}(0)=\left\langle k(0)\left|H_{I}^{(S)}(0)\right| j(0)\right\rangle \quad$ and $\quad 2 \pi \delta(\omega)=$ $\int_{-\infty}^{+\infty} d t e^{i \omega t}$. For discrete and normalized states, the $S$ matrix becomes infinite at the resonance due to the $\delta$ function, and the $T$ matrix is utilized instead [32]. However, for continuum states, the $\delta$ function describes the momentum distribution [33], namely, a single momentum component. For example, for a free electron, $\omega=\frac{\hbar k^{2}}{2 m}$, and

$$
\delta\left(\omega-\omega_{0}\right)=\frac{\delta\left(k-k_{0}\right)+\delta\left(k+k_{0}\right)}{|v|},
$$

where $v=\frac{\hbar}{m} k$. Note that the Jacobian factor $\frac{1}{v}=\frac{d k}{d \omega}$ becomes the density of states. For a one-dimensional continuum with $|k\rangle \equiv e^{i k z}$, such that $\left\langle k^{\prime} \mid k\right\rangle=2 \pi \delta\left(k-k^{\prime}\right)$, the $S$-matrix component becomes the final state in momentum space,

$$
\begin{aligned}
& \Psi_{f}(z)=S \Psi_{i}(z), \\
& \Psi_{f}(k)=\frac{1}{\sqrt{2 \pi}}\langle k|S| i\rangle=\frac{S_{k i}}{\sqrt{2 \pi}},
\end{aligned}
$$

using a unitary Fourier transform, and the final state in position space (in Schrödinger representation) becomes

$$
\begin{aligned}
\Psi_{f}^{(S)}(z, t) & =\frac{1}{\sqrt{2 \pi}} \int_{-\infty}^{+\infty} d k \Psi_{f}(k)|k\rangle e^{-i \omega t} \\
& =\frac{1}{2 \pi} \int_{-\infty}^{+\infty} d k S_{k i}|k\rangle e^{-i \omega t}
\end{aligned}
$$

Then, it follows, for a single energy component,

$$
\Psi_{f}^{(S)}(z, t)=-\frac{i}{\hbar} \frac{T_{k_{0} i}}{v_{0}}\left|k_{0}\right\rangle e^{-i \omega_{0} t}
$$

where we assumed $\delta_{k i}=0$.

For a perturbation that is finite in time, the $\delta$ function becomes a distribution that is broad and finite. We define the unnormalized Gaussian function as

$$
G(x, \sigma)=\exp \left[-\frac{x^{2}}{2 \sigma^{2}}\right]
$$

and the normalized Gaussian function as

$$
\widehat{g}(x, \sigma)=\frac{1}{\sqrt{2 \pi} \sigma} \exp \left[-\frac{x^{2}}{2 \sigma^{2}}\right],
$$


such that $\int_{-\infty}^{+\infty} d t G(t, \sigma) e^{i \omega t}=2 \pi \widehat{g}\left(\omega, \frac{1}{\sigma}\right)$. We define the time-dependent perturbation term as $\hat{V}_{s}(\mathbf{r}, t)=$ $\hat{V}_{s}(\mathbf{r}) \operatorname{Re}\left[e^{-i \omega_{s} t}\right] F(t)$, where the envelope function is given by $F(t)=\sqrt{G\left(t-\tau, \sigma_{e}\right)}=G\left(t-\tau, \sqrt{2} \sigma_{e}\right)=\exp \left[-\frac{(t-\tau)^{2}}{4 \sigma_{e}^{2}}\right]$, where $\sigma_{e}$ is the standard deviation width of the photoionization laser intensity profile. Note that we assume that photoionization is only significant at the surface, and the envelope function is at $z=0$ only. When the pump laser is also pulsed, $\alpha, \beta$, and $\gamma$, which are given by the amplitude of the electric field or the vector potential, also (slowly) vary in time. We define an envelope function for the electric field of the pump laser, $f(t)=\sqrt{G\left(t, \sigma_{p}\right)}=G\left(t, \sqrt{2} \sigma_{p}\right)=\exp \left[-\frac{t^{2}}{4 \sigma_{p}^{2}}\right]$, where $\sigma_{p}$ is the width of the pump laser intensity profile. Under the slowly varying envelope approximation $\gamma(t) \approx \gamma_{0} f(t)$, where $\gamma_{0}=\gamma(0)$, we obtain

$$
c_{n}(t)=\left(\frac{\gamma_{0}}{\left|\gamma_{0}\right|}\right)^{n} J_{n}\left(\left|\gamma_{0} f(t)\right|\right),
$$

and they need to be explicitly included in the time integration of the $S$ matrix. To this end, we define the composite envelope functions

$$
\begin{aligned}
\Gamma_{n}\left(t ; \tau ; \sigma_{e}, \sigma_{p}\right) & \equiv \frac{c_{n}(t)}{c_{n}(0)} F(t) \\
& =\frac{J_{n}\left(\left|\gamma_{0}\right| G\left(t, \sqrt{2} \sigma_{p}\right)\right)}{J_{n}\left(\left|\gamma_{0}\right|\right)} G\left(t-\tau, \sqrt{2} \sigma_{e}\right)(\mathrm{C} 10)
\end{aligned}
$$

such that $c_{n}(t) F(t)=c_{n}(0) \Gamma_{n}(t)$, and their nonunitary inverse Fourier transform

$$
2 \pi \widehat{\Gamma}_{n}\left(\omega ; \tau ; \frac{1}{\sigma_{e}}, \frac{1}{\sigma_{p}}\right) \equiv \int_{-\infty}^{+\infty} d t \Gamma_{n}\left(t ; \tau, \sigma_{e}, \sigma_{p}\right) e^{i \omega t} .
$$

Note that for a continuous pump beam $\left(\sigma_{p} \rightarrow \infty\right)$, the envelope function becomes a Gaussian function $\Gamma_{n}=G(t-$ $\left.\tau, \sqrt{2} \sigma_{e}\right)$, such that $\widehat{\Gamma}_{n}=\widehat{g}\left(\omega, \frac{1}{\sqrt{2} \sigma_{e}}\right) e^{i \omega \tau}$. When the probe laser is also continuous $\left(\sigma_{e} \rightarrow \infty\right)$, the latter becomes a delta function, $\widehat{g} \rightarrow \delta(\omega)$. Then, Eq. (9) becomes

$$
\begin{aligned}
S_{k b}^{B}-1 & =-\frac{i}{\hbar} \int_{-\infty}^{+\infty} d t\left\langle\Psi_{n}^{V}(t)\left|\hat{V}_{s}(\mathbf{r}, t)\right| \Psi_{b}^{F}(t)\right\rangle \\
& =-\frac{i}{\hbar}\left\langle k\left|\frac{\hat{V}_{s}}{2}\right| b\right\rangle \int_{-\infty}^{+\infty} d t \sum_{n} c_{n}(t) F(t) e^{i\left(\omega-\omega_{0}-n \omega_{p}\right) t} \\
& =-\frac{i}{\hbar} H_{k b} \sum_{n} c_{n}(0) \int_{-\infty}^{+\infty} d t \Gamma_{n}(t) e^{i\left(\omega-\omega_{0}-n \omega_{p}\right) t} \\
& =-\frac{i}{\hbar} H_{k b} \sum_{n} c_{n}(0) 2 \pi \widehat{\Gamma}_{n}\left(\omega-\omega_{n}\right),
\end{aligned}
$$

where we define

$$
T_{n b}^{(0)} \equiv H_{n b} c_{n}(0)
$$

Then the Schrödinger representation becomes

$$
\begin{aligned}
\Psi_{f}^{B}(z, t) & =\frac{1}{2 \pi} \int_{-\infty}^{+\infty} d k S_{k i}^{B} \Psi_{k}^{V}(t) \\
& =-\frac{i}{\hbar} \sum_{n} T_{n b}^{(0)} \int d k \widehat{\Gamma}_{n}\left(\omega-\omega_{n}\right) \Psi_{k}^{V}(t)
\end{aligned}
$$

$$
\begin{aligned}
& \approx-\frac{i}{\hbar} \sum_{n} \frac{T_{n b}^{(0)}}{v_{n}} \int d k \widehat{\Gamma}_{n}\left(k-k_{n}\right) \Psi_{k}^{V}(t) \\
& \approx-\frac{i}{\hbar} \sum_{n} \frac{T_{n b}^{(0)}}{v_{n}} \Gamma_{n}^{(-)}\left(z-v_{n} t\right) \Psi_{n}^{V}(t),
\end{aligned}
$$

where $\widehat{\Gamma}_{n}\left(k-k_{n}\right)=\widehat{\Gamma}_{n}\left(k-k_{n} ; v_{n} \tau ; \frac{1}{v_{n} \sigma_{e}}, \frac{1}{v_{n} \sigma_{p}}\right)$, and $\Gamma_{n}^{(-)}(z-$ $\left.v_{n} t\right)=\Gamma_{n}\left(-z+v_{n} t ; v_{n} \tau ; v_{n} \sigma_{e}, v_{n} \sigma_{p}\right)$ is the double inverse Fourier transform of $\Gamma_{n}$. Note that $\Gamma_{n}^{(-)}(\xi)=\Gamma_{n}(-\xi)$, because $\mathcal{F}\{f(\xi)\}=\mathcal{F}^{-1}\{f(-\xi)\}$. We approximated that the Volkov phase in $\Psi_{k}^{V}=e^{i\left(k z-\omega t-\alpha \sin \omega_{p} t\right)}$ remains constant in $k$ integration, and we ignored the wave-packet dispersion by $\omega \approx \omega_{n}+v_{n}\left(k-k_{n}\right)$. Also note that $\omega_{n, j}=\frac{\hbar}{2 m} k_{n}^{2}+j \omega_{p}$ and its dispersion relation and the group velocity are independent of $j \omega_{p}$, namely, $\frac{\partial \omega_{n, j}}{\partial k_{n}}=v_{n}$, for each $n$. Equation (C14) shows that the photoelectron temporal profile follows that of the composite envelope function. The wave packet in the $n$th state (after collapse of the Volkov states) is

$$
\Psi_{n}(z, t)=-\frac{i}{\hbar} \frac{T_{n b}^{(0)}}{v_{n}} \Gamma_{n}^{(-)}\left(z-v_{n} t\right) e^{i\left(k_{n} z-\omega_{n} t\right)},
$$

and the intensity (probability density) is given by

$$
\begin{aligned}
I_{n}(z, t) & =\left|\frac{T_{n b}^{(0)}}{\hbar v_{n}} \Gamma_{n}^{(-)}\left(z-v_{n} t\right)\right|^{2} \\
& =\left|\frac{H_{n b}}{\hbar v_{n}} c_{n}(0) \Gamma_{n}^{(-)}\left(z-v_{n} t\right)\right|^{2} \\
& =\left|\frac{H_{n b}}{\hbar v_{n}} c_{n}\left(t-\frac{z}{v_{n}}\right) F\left(t-\frac{z}{v_{n}}\right)\right|^{2} \\
& =\left|\frac{H_{n b}}{\hbar v_{n}} c_{n}\left(t-\frac{z}{v_{n}}\right)\right|^{2} G\left(t-\tau-\frac{z}{v_{n}}, \sigma_{e}\right) \\
& \approx I_{00}\left|c_{n}\left(t-\frac{z}{v_{n}}\right)\right|^{2} G\left(t-\tau-\frac{z}{v_{n}}, \sigma_{e}\right),
\end{aligned}
$$

where we approximated $\frac{H_{n b}}{\hbar v_{n}} \approx \frac{H_{0 b}}{\hbar v_{0}} \equiv I_{00}$, such that $I_{00}$ is the continuous photoemission intensity with no pump laser. Then, the probability is given by

$$
\begin{aligned}
P_{n} & =\int_{0}^{+\infty} d z I_{n}(z, t) \\
& =I_{00} v_{n} \int_{-\infty}^{t} d \xi\left|c_{n}(\xi) F(\xi)\right|^{2},
\end{aligned}
$$

where $\xi=t-\frac{z}{v_{n}}$. Since $\sum_{n}\left|c_{n}(\xi)\right|^{2}=1$ at any $\xi$, the total photoemission is

$$
\begin{aligned}
\sum_{n} P_{n} & =I_{00} \sum_{n} v_{n} \int_{-\infty}^{t} d \xi\left|c_{n}(\xi) F(\xi)\right|^{2} \\
& \approx I_{00} v_{0} \sum_{n} \int_{-\infty}^{t} d \xi\left|c_{n}(\xi) F(\xi)\right|^{2} \\
& =I_{00} v_{0} \int_{-\infty}^{t} d \xi|F(\xi)|^{2} \\
& \rightarrow I_{00} v_{0} \sqrt{2 \pi} \sigma_{e} \quad\left(\text { for } t-\tau \gg \sigma_{e}\right) .
\end{aligned}
$$


The normalized population (fraction) becomes

$$
\begin{aligned}
Q_{n} & =\frac{P_{n}}{\sum_{n} P_{n}} \approx \frac{1}{\sqrt{2 \pi} \sigma_{e}} \int_{-\infty}^{t} d \xi\left|c_{n}(\xi) F(\xi)\right|^{2} \\
& =\int_{-\infty}^{t} d \xi\left|c_{n}(\xi)\right|^{2} \frac{1}{\sqrt{2 \pi} \sigma_{e}} G\left(\xi-\tau, \sigma_{e}\right) \\
& =\int_{-\infty}^{t} d \xi\left|c_{n}(\xi)\right|^{2} \widehat{g}\left(\xi-\tau, \sigma_{e}\right),
\end{aligned}
$$

which shows that the photoemission intensity in the continuous case can be treated as an instantaneous intensity, such that it allows us to use a semiclassical approximation to perform a profile averaging to obtain the total population.

However, the integration in Eq. (C19) is not analytically obtainable to our best knowledge, particularly because the Bessel function is oscillatory for a large argument. Nevertheless, for a weak interaction $(|\gamma| \ll \sqrt{n+1}), J_{n}(|\gamma|) \rightarrow \frac{1}{n !}\left(\frac{|\gamma|}{2}\right)^{n}$ for $n>0$, we may further approximate $J_{n}(f|\gamma|) \approx f^{n} J_{n}(|\gamma|)$. Then, we simply have $\Gamma_{n} \approx f^{n} F$, and obtain

$$
\begin{aligned}
\left|\Gamma_{n}^{(-)}\right|^{2} & =G\left(\xi, \frac{\sigma_{p}}{\sqrt{n}}\right) G\left(\xi-\tau, \sigma_{e}\right) \\
& =G\left(\tau, \Sigma_{n}\right) G\left(\xi-\tau_{n}, \Omega_{n}\right),
\end{aligned}
$$

where $\Sigma_{n}^{2}=\sigma_{e}^{2}+n^{-1} \sigma_{p}^{2}, \Omega_{n}^{-2}=\sigma_{e}^{-2}+n \sigma_{p}^{-2}$, and $\tau_{n}=\tau \frac{\sigma_{e}}{\Sigma_{n}}$ and therefore

$$
Q_{n} \rightarrow Q_{n}^{(0)} G\left(\tau, \Sigma_{n}\right) \frac{\Omega_{n}}{\sigma_{e}}=Q_{n}^{(0)} \frac{G\left(\tau, \Sigma_{n}\right)}{\sqrt{1+n \frac{\sigma_{e}^{2}}{\sigma_{p}^{2}}}},
$$

where $Q_{n}^{(0)}$ is when $\sigma_{p} \rightarrow \infty$. After a similar treatment for the spatial overlap, we obtain

$$
Q_{n} \rightarrow Q_{n}^{(0)} \prod_{\xi=t, x, y} \frac{G\left(\Delta \xi, \Sigma_{n, \xi}\right)}{\sqrt{1+n \frac{\sigma_{e, \xi}^{2}}{\sigma_{p, \xi}^{2}}}}
$$

where $\Sigma_{n, \xi}=\sqrt{\sigma_{e, \xi}^{2}+\frac{1}{n} \sigma_{p, \xi}^{2}}$.

\section{APPENDIX D: SCALING FACTOR FOR PROFILE AVERAGING}

We define a scaling factor $\rho_{n}$ such that $Q_{n}=Q_{n}^{(0)}\left(\gamma_{0} \rho_{n}\right)$. Since we approximated $Q_{n}^{(0)}\left(\gamma_{0} \rho_{n}\right) \approx\left(\rho_{n}\right)^{2 n} Q_{n}^{(0)}\left(\gamma_{0}\right)$ for a weak interaction, we obtain

$$
\rho_{n} \approx\left(\prod_{\xi=t, x, y} \frac{G\left(\Delta \xi, \Sigma_{n, \xi}\right)}{\sqrt{1+n \frac{\sigma_{e, \xi}^{2}}{\sigma_{p, \xi}^{2}}}}\right)^{\frac{1}{2 n}} .
$$

Then, for the three-dimensional case (time and space dimensions) with a perfect overlap $\left(\sigma_{e, \xi}=\sigma_{p, \xi}\right.$ and $\left.\Delta \xi=0\right)$, $Q_{1}^{(0)}\left(\gamma_{0} \rho_{1}\right)=\left|J_{1}\left(\gamma_{0} \rho_{1}\right)\right|^{2}$ with a scale factor of $\rho_{1}=0.5946$ approximately gives the population for $n=1$ with profile averaging.
[1] G. Floquet, Ann. Sci. Ec. Norm. Sup. Sér. 2 12, 47 (1883).

[2] J. H. Shirley, Phys. Rev. 138, B979 (1965).

[3] H. G. Muller, H. B. van Linden van den Heuvell, and M. J. van der Wiel, J. Phys. B: At. Mol. Phys. 19, L733 (1986).

[4] T. E. Glover, R. W. Schoenlein, A. H. Chin, and C. V. Shank, Phys. Rev. Lett. 76, 2468 (1996).

[5] L. B. Madsen, Am. J. Phys. 73, 57 (2005).

[6] J. C. Baggesen and L. B. Madsen, Phys. Rev. A 78, 032903 (2008).

[7] M. A. Acuña and M. S. Gravielle, Phys. Rev. A 83, 032904 (2011).

[8] G. Saathoff, L. Miaja-Avila, M. Aeschlimann, M. M. Murnane, and H. C. Kapteyn, Phys. Rev. A 77, 022903 (2008).

[9] D. M. Wolkow, Z. Phys. 94, 250 (1935).

[10] Y. H. Wang, H. Steinberg, P. Jarillo-Herrero, and N. Gedik, Science 342, 453 (2013).

[11] Y. Zhou and M. W. Wu, Phys. Rev. B 83, 245436 (2011).

[12] H. R. Reiss, Phys. Rev. A 22, 1786 (1980).

[13] $A_{1 z}$ in Eq. (3) is in phase with $A_{2 x}$ in Eq. (1).

[14] When the ponderomotive term is explicitly considered, the Volkov state is given by the generalized Bessel function (see Ref. [12]).

[15] J. Bergou and S. Varró, J. Phys. A: Math. Gen. 13, 3553 (1980).
[16] For $\mathbf{A}(t)=\operatorname{Re}\left[\hat{\mathbf{z}} \tilde{A}_{1} e^{-i \omega_{p} t}\right]$, we obtain $a_{j} \equiv\left(\frac{\tilde{\alpha}}{|\tilde{\alpha}|}\right)^{j} \mathbf{J}_{j}(|\tilde{\alpha}|)$ where $\tilde{\alpha}=\frac{e \mathbf{v} \cdot \tilde{\mathbf{A}}_{1}}{\hbar \omega_{p}}$.

[17] Equation (5) is an approximation since the Volkov solution is not an eigenstate for the bound electron Hamiltonian, nor is the bound state an eigenstate for the free electron Hamiltonian.

[18] Here, we ignore autoionization pathways and only consider direct ionization.

[19] $\delta(\omega) \equiv \frac{1}{2 \pi} \int_{-\infty}^{+\infty} d t \cos (\omega t)$.

[20] Note that $\alpha$ also slightly depends on $n$ via $k_{n}$.

[21] Note that in LAES, the (initial) incident and (final) outgoing electrons interact with the same light, resulting in always destructive interference of Volkov expansions, such that $c_{n}=$ $J_{n}\left(\alpha_{i}-\alpha_{f}\right)$ where $\alpha_{j}=\frac{e \mathbf{v}_{j} \cdot \mathbf{A}_{j}}{\hbar \omega_{p}}$ for $j=i, f$. Similarly, for Floquet-LAPE, both $\tilde{\alpha}$ and $\tilde{\beta} \equiv \beta^{\|} e^{i \phi \|}$ can be consistently expressed as $\frac{e \mathbf{v}_{j} \cdot \tilde{\mathbf{A}}_{j}}{\hbar \omega_{p}}$ for $j=1,2$, and $c_{n}$ is determined by $\gamma=\lambda \tilde{\beta}-\alpha$.

[22] N. M. Kroll and K. M. Watson, Phys. Rev. A 8, 804 (1973).

[23] N. K. Rahman, Phys. Rev. A 10, 440 (1974).

[24] I. S. Gradshteyn and I. M. Ryzhik, Table of Integrals, Series and Products, 5th ed. (Academic, New York, 1994).

[25] J. D. Jackson, Classical Electrodynamics (Wiley, New York, 1975). 
[26] $\mathrm{UV}$ photon energy is $6.2 \mathrm{eV}$, and the work function of $\mathrm{Bi}_{2} \mathrm{Se}_{3}$ is estimated to be $5.5 \mathrm{eV}$.

[27] In Ref. [10], the authors observed the momentum dependence of the side band intensity that is stronger for the perpendicular momentum than the parallel, and argue that it is the opposite to LAPE theory. While the surface bound electron only possesses 2D velocity $\left(v_{F}\right)$ and associated momentum $\left(k_{x}\right.$ and $\left.k_{y}\right)$, the photoelectron acquires a significant $k_{z}$ component from the ionization laser, and $\alpha$ is dominated by the $z$ components of the free electron velocity and the IR laser. Therefore, the LAPE efficiency by itself should show very little dependence on the initial (bound) momentum, which is contrary to the authors' argument against LAPE. Furthermore, the simulation (see Fig. 3) predicts that Floquet side band intensity by itself is much smaller for the perpendicular polarization than the parallel polarization, especially for high- $k$ components $\left(\left|k_{y}\right|>0.03 \AA^{-1}\right)$, which contradicts their observed momentum dependence.
[28] This coupling makes the unperturbed photoelectron energy spectrum severely asymmetric, such that it hinders the absolute comparison between $+k_{x}$ and $-k_{x}$ spectra where the FloquetVolkov interference is expected to be most significant. Rather, the relative intensity of the side bands with respect to their main bands $(n=0)$ should be compared.

[29] The authors in Ref. [10] did not quantify the ratio, but only qualitatively stated the relative intensity.

[30] See Supplemental Material at http://link.aps.org/supplemental/ 10.1103/PhysRevA.90.013420 for a side-by-side comparison.

[31] J. J. Sakurai, Advanced Quantum Mechanics (Addison-Wesley, Reading, 1967).

[32] Joachain explains that the $\delta$ function is of no concern because one eventually consider transitions into a group of states in the continuum; see C. J. Joachain, Quantum Collision Theory (North-Holland, Amsterdam, 1975), p. 359.

[33] T. Kopaleishvili, Collision Theory (A Short Course) (World Scientific, Singapore, 1995). 